\title{
A Farey Tail
}

\author{
Patrice Philippon
}

The Garden of Visibles and the Farey Graph

The garden of visibles in the lattice $\mathbf{Z}^{2}$ (i.e., of points visible from the origin; cf. [2, page 29]) arises when one plants a tree at each point with relatively prime coordinates. The rays issuing from these visible points and pointing away from the origin cover all the hidden points of $\mathbf{Z}^{2}$ (i.e., the points that are not visible from the origin because they lie behind a visible one). We complete these hidden rays to an infinite graph, which we dub the hidden graph, by linking each visible point to the lower and upper neighboring rays with vertical line segments (and of course declaring the intersection points to be vertices).

In Figure 1 the origin is represented by the blue dot, and we have sketched a sector of the hidden graph. The complete graph is obtained as the union of images of this sector under the transformations $(x, y) \mapsto( \pm x, y+n x), n \in \mathbf{Z}$, plus eventually the two isolated half-lines $\{0\} \times[1,+\infty[$ and $\{0\} \times]-\infty,-1]$. We will call any vertex coinciding with a visible point an extremal vertex of the hidden graph. These are the main vertices from which all the rays (slanted edges) of the graph stem.

For a better view it is adequate to straighten the hidden graph through the map $[1, \infty[\times \mathbf{R} \rightarrow$ $\left[1, \infty\left[\times \mathbf{R},(x, y) \mapsto\left(x, \frac{y}{x}\right)\right.\right.$, thus obtaining the Farey tail, where the radial edges become parallel to the first horizontal axis and the edges parallel to the second vertical axis keep the same direction.

Thus, the Farey tail is the graph whose extremal vertices are the points $\left(q, \frac{p}{q}\right)$, where $\frac{p}{q}$ runs

Patrice Philippon is Directeur de Recherche at CNRS, working at the Institut de Mathématiques de Jussieu in Paris. His email address is pph@math. jussieu.fr.

The author warmly thanks Dale Brownawell for his careful reading of this text and his help in straightening out many (linguistic or not) obscurities. He also thanks the editors of the Notices for their encouragement and work in order to publish the article.

DOI: http://dx.doi.org/10.1090/noti860

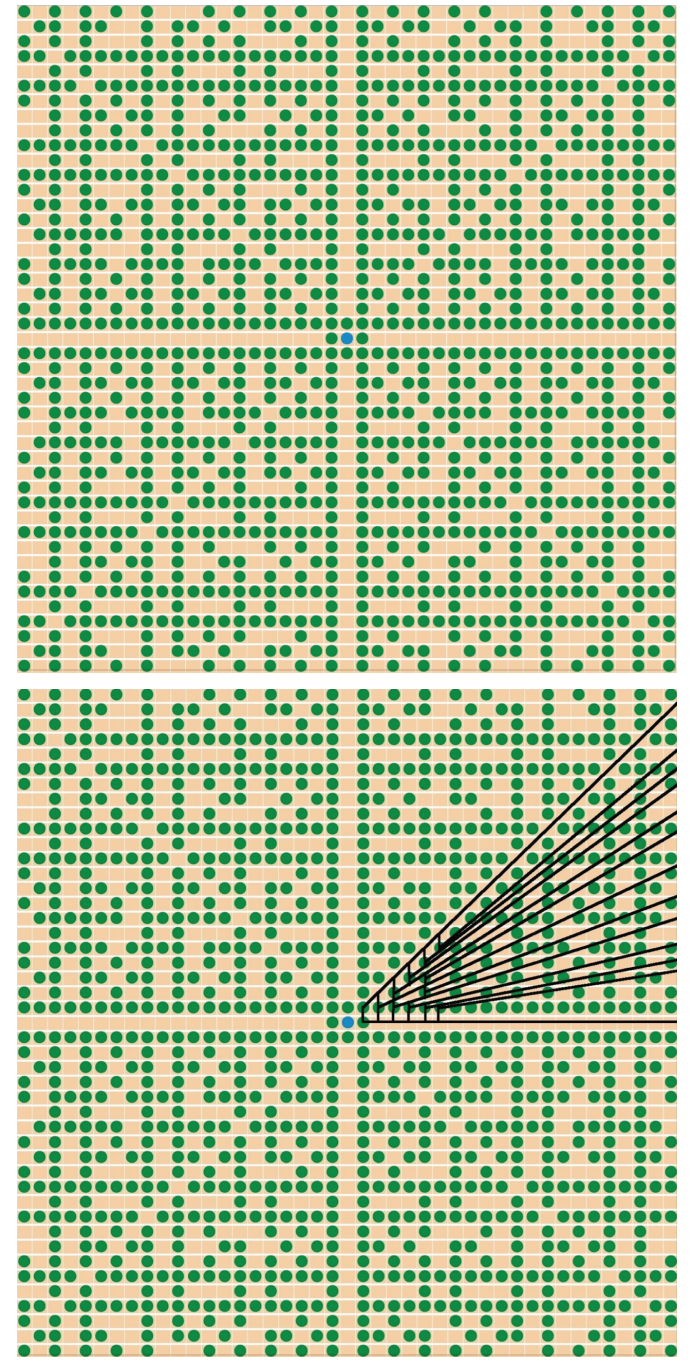

Figure 1. The garden of visibles and the hidden graph.

over the set of rational numbers (written in reduced form), and the edges are the horizontal half-lines $\left(u, \frac{p}{q}\right), u \geq q$, together with the vertical segments linking each extremal vertex to vertices on the 
neighboring edges. The graph is periodic through vertical translation by 1 and it is symmetric with respect to the line of ordinate $\frac{1}{2}$. Figure 2 shows only the stripe $[1, \infty[\times[0,1]$, corresponding to the sector of the hidden graph already sketched in Figure 1.

Let's recall that for $n \in \mathbf{N}^{*}$ the $n$-th Farey sequence, denoted $\mathcal{F}_{n}$, is the sequence, in increasing order, of rational numbers between 0 and 1 , the denominators of which are at most $n$ : $\mathcal{F}_{1}=0,1 ; \mathcal{F}_{2}=0, \frac{1}{2}, 1 ; \mathcal{F}_{3}=0, \frac{1}{3}, \frac{1}{2}, \frac{2}{3}, 1 \ldots ;$ see $[4$, §1 \& 2] or [2, chap. 3], for example. Beware that in [4] the $n$-th Farey sequence, $n \in \mathbf{N}$, is extended to all rational numbers with denominator and absolute value of numerator at most $n$, furthermore including both "infinities" $\frac{-1}{0}$ and $\frac{1}{0}$ at the extremes: $\mathcal{F}_{0}^{\prime}=\frac{-1}{0}, \frac{0}{1}, \frac{1}{0} ; \mathcal{F}_{1}^{\prime}=\frac{-1}{0}, \frac{-1}{1}, \frac{0}{1}, \frac{1}{1}, \frac{1}{0}$; $\mathcal{F}_{2}^{\prime}=\frac{-1}{0}, \frac{-2}{1}, \frac{-1}{1}, \frac{-1}{2}, \frac{0}{1}, \frac{1}{2}, \frac{1}{1}, \frac{2}{1}, \frac{1}{0} \ldots$. We recover the Farey sequence $\mathcal{F}_{n}$ on the Farey tail depicted in Figure 2, for example, as the sequence of ordinates of horizontal edges of the Farey graph encountered when going up the vertical line of abscissa $n$ (or of any abscissa at least $n$ and strictly less than $n+1$ ).

The Farey comb (Figure 3) is the horizontal contraction of the preceding graph through the transformation $(u, v) \longmapsto\left(\frac{u-1}{u}, v\right)$. As for the Farey tail, we show only the part of the graph in the square $[0,1[\times[0,1]$; the complete comb is the union of translates of this part by the points $(0, n)$, $n \in \mathbf{Z}$.

Finally, the Farey eye is obtained from the previous graph as an image under the exponential map $(s, t) \longmapsto s \mathrm{e}^{2 \mathrm{i} \pi t}$ in the unit disc $D(0,1)$ of the complex plane. This transformation takes care of the periodicity of the Farey comb through entire vertical translations. In Figure 4 the Farey eye as just introduced is on the left; the right picture is its symmetric reflection (either through the origin or the vertical axis, since the eye is itself symmetric with respect to the horizontal axis).

To sum up, here are the transformations between the different representations of the Farey graph: ${ }^{1}$

$$
\begin{aligned}
& {[1,+\infty[\times \mathbf{R} \rightarrow[1,+\infty[\times[0,1[\rightarrow[0,1] \times[0,1[\rightarrow \quad D(0,1)} \\
& (x, y) \mapsto\left(x,\left\{\frac{y}{x}\right\}\right) \mapsto\left(\frac{x-1}{x},\left\{\frac{y}{x}\right\}\right) \mapsto\left(\frac{x-1}{x}, 2 \pi\left\{\frac{y}{x}\right\}\right) \\
& (u, v) \quad \hookleftarrow \quad\left(\frac{u-1}{u}, v\right) \mapsto\left(\frac{u-1}{u}, 2 \pi v\right) \\
& (s, t) \quad \mapsto \quad(s, 2 \pi t) \\
& (\rho, \theta)
\end{aligned}
$$

\footnotetext{
${ }^{1}$ We denote by $\{\star\}$ the number in the interval $[0,1[$ congruent to $\star$ modulo Z. For a visual summary of these transformations and more, visit: http://www.math. jussieu.fr/ pph/ and double-click on the black screen there.
}

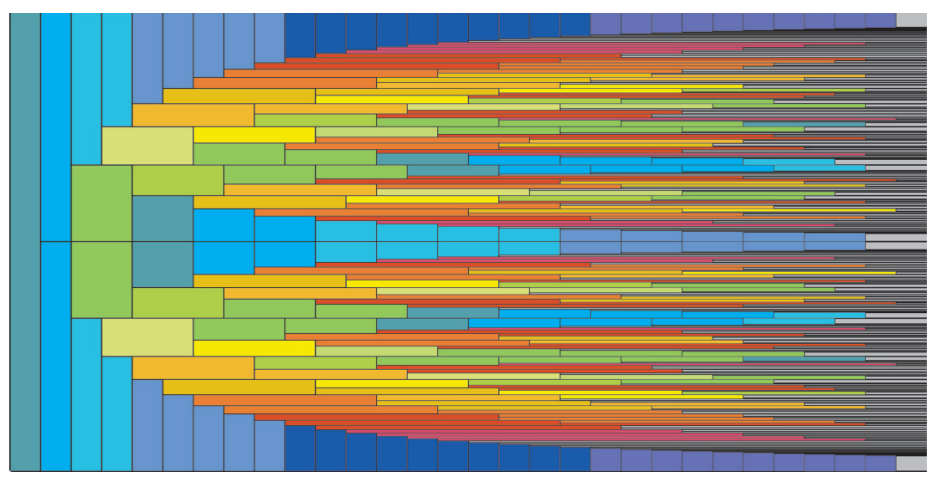

Figure 2. The Farey tail.
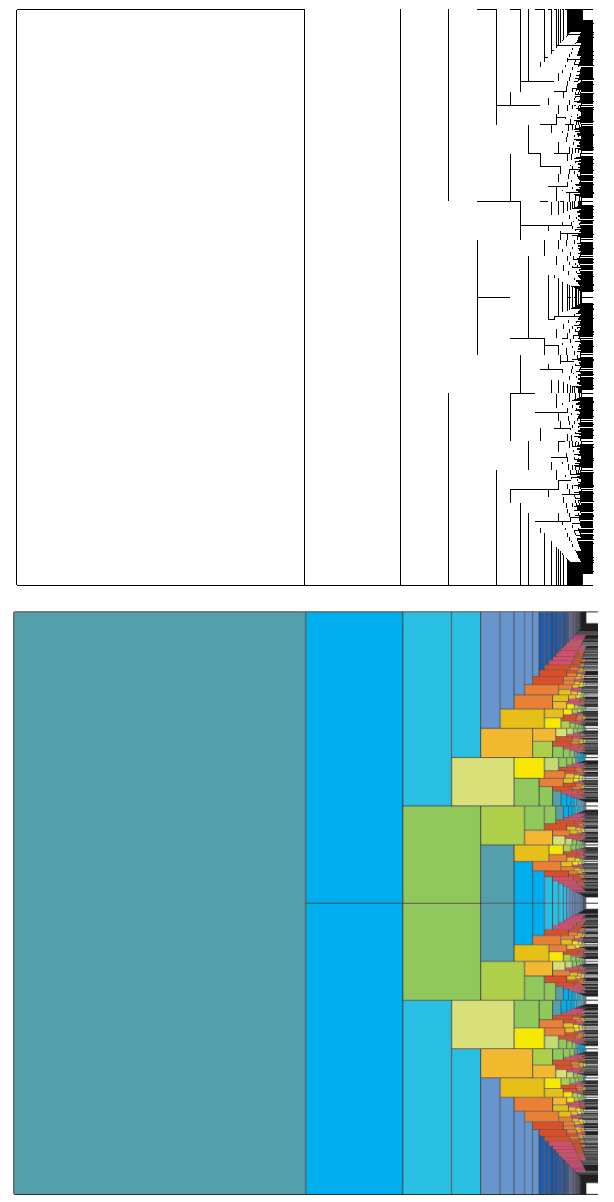

Figure 3. The Farey comb.

The first three systems of coordinates are Cartesian, and the last one is polar. ${ }^{2}$

As one might guess from Figures 2-4, the Farey graph is a genuine fractal object. Indeed, the fractal dimension (also called Mandelbrot or Kolmogorov dimension) of the Farey comb or of the Farey eye

\footnotetext{
${ }^{2}$ The reader may be interested in checking that lines in the garden of visibles (except the vertical axis) are transformed into lines in the Farey comb.
} 


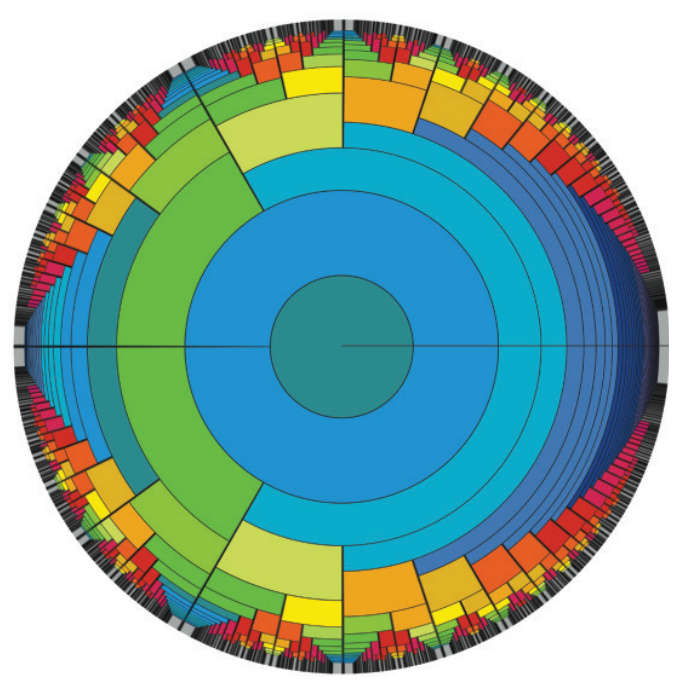

The Farey eye.

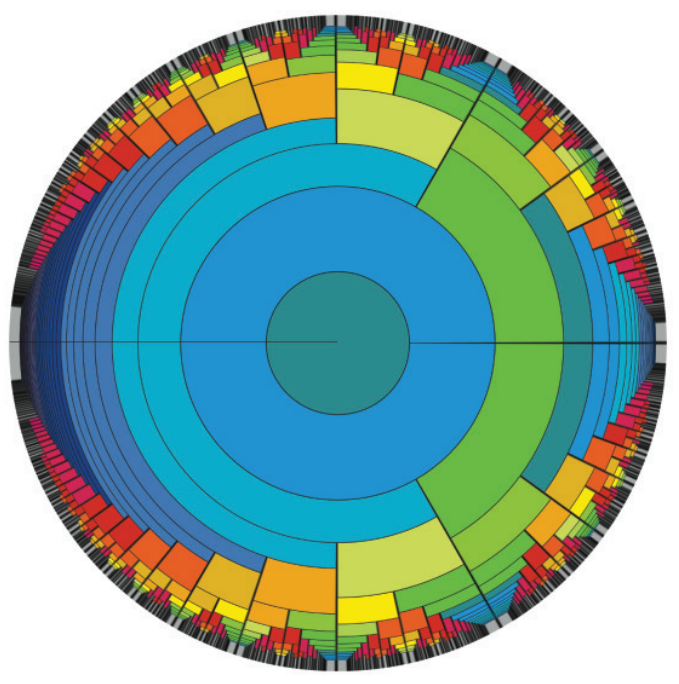

The symmetrical Farey eye.

Figure 4

as subsets of $\mathbf{R}^{2}$ (that is, of the image graphs in the square or in the unit disc) is equal to $3 / 2$, a true fraction. ${ }^{3}$

\section{Paths and Best Approximations}

Let's orient the edges of the Farey graph so that in the Farey tail, for example, one can move along the horizontal edges from the left to the right and on the vertical edges toward the vertex (dubbed extremal vertex in the previous section) of the unique horizontal edge stemming from this vertical edge and running to the right.

A path in the Farey tail can therefore be described by a sequence of fractions $\frac{p_{0}}{q_{0}}, \frac{p_{1}}{q_{1}}, \ldots$ corresponding to the different horizontal edges it takes. We note that, in fact, one can enter such a horizontal edge only from its extremal vertex, where the only two vertical edges oriented toward this horizontal edge end.

\section{One-Sided Approximations}

We introduce the following area form, expressed in the different representations of the Farey graph described in the previous section:

$$
\frac{d x \wedge d y}{x} \leftrightarrow d u \wedge d v \leftrightarrow \frac{d s \wedge d t}{(1-s)^{2}} \leftrightarrow \frac{d \rho \wedge d \theta}{2 \pi(1-\rho)^{2}} .
$$

We associate to a real number $\xi \in[0,1[$ the ray in the Farey eye, the angle of which is $2 \pi \xi$, and we consider the path in the Farey graph minimizing the area between this ray and the path, computed with the area form above. Alternatively, it is the path in the Farey eye for which the radial edges

\footnotetext{
${ }^{3}$ Computation of this fractal dimension can be found in: P. Philippon, Un oeil et Farey, http://ha1. archives-ouvertes.fr/ha1-00488471 (02/06/2010).
}

cut a minimal angle on the unit circle, with the direction determined by $\xi$. If $\frac{p_{0}}{q_{0}}, \frac{p_{1}}{q_{1}}, \ldots$ is the sequence of fractions describing this path, the area is given by the formula (for the computation, one may prefer to go back to the Farey tail)

$$
\sum_{k=0}^{\infty}\left(q_{k+1}-q_{k}\right)\left|\xi-\frac{p_{k}}{q_{k}}\right| .
$$

Therefore, it is minimal when each irreducible fraction $\frac{p_{k}}{q_{k}}$ represents the rational number of denominator $\leq q_{k}$ closest to $\xi$. Thus, the path under consideration is described by the sequence of fractions giving the best approximations of type 1 of $\xi$, that is, satisfying for all $k \in \mathbf{N}$ :

$$
\begin{aligned}
& \forall(p, q) \in \mathbf{Z} \times \mathbf{N}^{*}, \\
& q \leq q_{k}, \frac{p}{q} \neq \frac{p_{k}}{q_{k}}, \\
& \left|\xi-\frac{p}{q}\right|>\left|\xi-\frac{p_{k}}{q_{k}}\right| .
\end{aligned}
$$

We remark that since two consecutive elements in the Farey sequence $\mathcal{F}_{n}, n \in \mathbf{N}, n>1$, cannot have the same denominator (cf. [2, Thm. 31]), condition (2) determines uniquely the sequence of best approximations $\frac{p_{k}}{q_{k}}$ with $q_{k}>1$. Furthermore, when $q_{k}=1$ there is ambiguity determining the best integral approximation only for the half integers $\xi \in \frac{1}{2}+\mathbf{N}$. Therefore, to each real number $\xi$ (not a half integer) is associated a definite path in the Farey eye which then becomes an "approximoscope"; see Figure 5, where, following the white lightning, one reads the sequence of best approximations of type 1 of $\frac{9}{38}: 0, \frac{1}{3}, \frac{1}{4}, \frac{3}{13}, \frac{4}{17}, \frac{5}{21}$, $\frac{9}{38}$. 


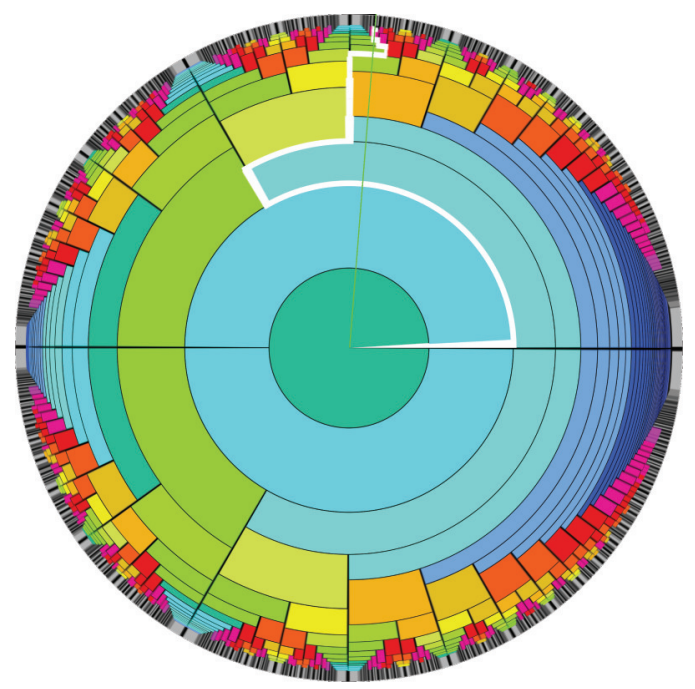

Figure 5. The approximoscope set at $\xi=\frac{9}{38}$ (green ray).

We note that the convergents of the regular continued fraction of $\xi$ are some best approximations of type 1, in the sense of (2) (cf. [2, Thm. 181]), except possibly for the first one, since the first best approximation $\frac{p_{0}}{q_{0}}$ of $\xi$ (with denominator $q_{0}=1$ ) is the integer closest to $\xi$, not its integer part (with the ambiguity already mentioned for half integers $\xi \in \frac{1}{2}+\mathrm{N}$ ). But these convergents of the regular continued fraction do not give all the best approximations of type 1 . They give all the best approximations of type 0 , in the stronger sense (cf. [2, Thm. 182]):

$$
\begin{aligned}
& \forall(p, q) \in \mathbf{Z} \times \mathbf{N}^{*}, \\
& q \leq q_{k}, \frac{p}{q} \neq \frac{p_{k}}{q_{k}}, \\
& |q \xi-p|>\left|q_{k} \xi-p_{k}\right| .
\end{aligned}
$$

The quantity $|q \xi-p|$ can be visualized in the Farey comb as the absolute value of the slope of the line linking the point $(1, \xi)$ to the point $\left(1-\frac{1}{q}, \frac{p}{q}\right)$. Therefore, the approximations of type 0 are represented by the points $\left(1-\frac{1}{q}, \frac{p}{q}\right)$ such that the rhomboid defined by the equations $0 \leq x<1-\frac{1}{q}$ and $|\xi-y| \leq|q \xi-p| .(1-x)$ does not contain any vertex of the Farey comb.

This leads to Klein's geometric interpretation, according to which these best approximations of type 0 can be detected as follows. From the left split the horizontal line of ordinate $\xi$ in two and pull the extremity of each resulting string downwards for the lower string and diagonally left upwards for the upper string (see Figure 6, where $\xi=\frac{9}{38}$ is the ordinate of the green horizontal line). Suppose these rubber strings cannot cross the horizontal edges of the graph; then they shape polygonal lines spanned on the vertices of some horizontal edges of the graph. (In Figure 6 these two polygonal lines are drawn in black, while the white line is the lightning of best approximations of type 1 discussed previously, the image of which in the Farey eye has already been shown in Figure 5.) The corners of these polygonal lines correspond alternatively to the upper and lower best approximations of type 0 of $\xi$. Furthermore, between two corners, the number of horizontal edges touched at their vertices by the polygonal line is the corresponding partial quotient of the expansion in regular continued fraction ${ }^{4}$ of $\xi$ minus 1.

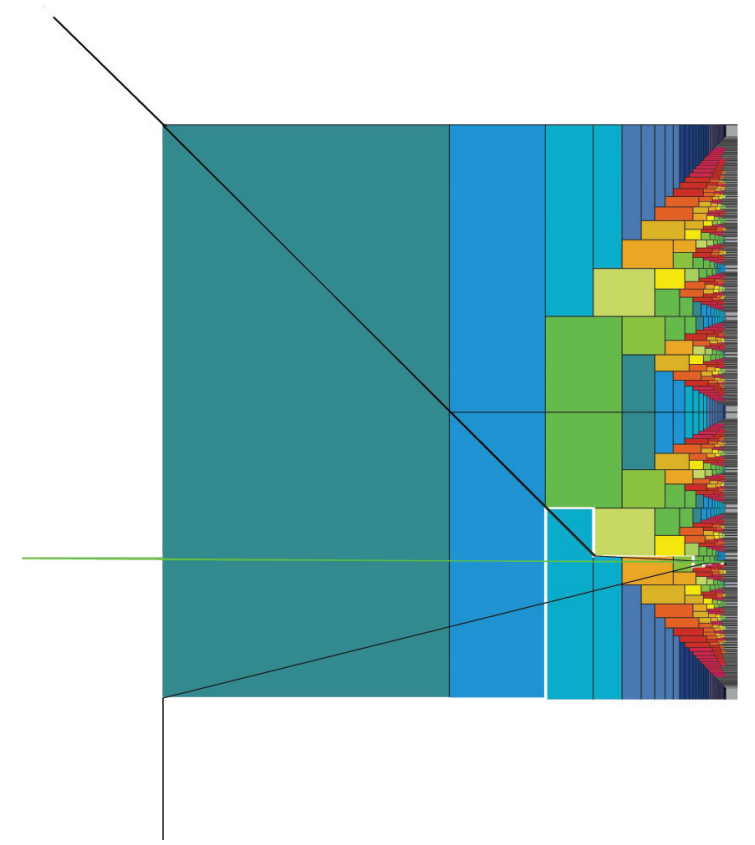

Figure 6. Farey comb with lightning and strings spanned at $\xi=\frac{9}{38}$.

Example 1. 1) The convergents of the regular continued fraction of $\frac{1}{4}$ are 0 and $\frac{1}{4}$, the best approximations of type 0 , whereas the sequence of best approximations of type 1 is $0, \frac{1}{3}, \frac{1}{4}$.

2) In Figure 6 the upper and lower black lines are composed of two and three segments respectively. For the lower one, the first segment goes upwards to the vertex $(0,0)$; the second segment from $(0,0)$ to $\left(\frac{16}{17}, \frac{4}{17}\right)$, touching the intermediate vertices $\left(\frac{4}{5}, \frac{1}{5}\right),\left(\frac{8}{9}, \frac{2}{9}\right),\left(\frac{12}{13}, \frac{3}{13}\right)$; and the third lower segment goes directly from $\left(\frac{16}{17}, \frac{4}{17}\right)$ to $\left(\frac{37}{38}, \frac{9}{38}\right)$. The first segment in the upper line goes from

\footnotetext{
${ }^{4}$ More on continued fraction expansions will come in the next section. See in particular the beginning of the subsection "Sequences of Approximations" to identify the intermediate vertices with the intermediate convergents, first in the garden of visibles, then in the Farey comb, thanks to the hint in footnote 2.
} 
the upper left corner down to $\left(\frac{3}{4}, \frac{1}{4}\right)$, touching the intermediate vertices $(0,1),\left(\frac{1}{2}, \frac{1}{2}\right),\left(\frac{2}{3}, \frac{1}{3}\right)$; and the second segment goes from $\left(\frac{3}{4}, \frac{1}{4}\right)$ to $\left(\frac{37}{38}, \frac{9}{38}\right)$, touching the intermediate vertex $\left(\frac{20}{21}, \frac{5}{21}\right)$. The sequence of corners is therefore $(0,0),\left(\frac{3}{4}, \frac{1}{4}\right),\left(\frac{16}{17}, \frac{4}{17}\right)$, and $\left(\frac{37}{38}, \frac{9}{38}\right)$, giving the sequence of best approximations of type 0 of $\frac{9}{38}: 0, \frac{1}{4}, \frac{4}{17}, \frac{9}{38}$. Counting the number of intermediate vertices on each segment, one gets the expansion in regular continued fractions: $\frac{9}{38}=[0,4,4,2]$ (the last segment of the lower string is ignored, since the number is reached from above, but for irrational numbers the sequence of segments continues indefinitely).

\section{Two-Sided Approximations}

In the same notation as above, starting from the pair of integers $\left(\frac{[\xi]}{1}, \frac{[\xi]+1}{1}\right)$ framing the real number $\xi$, one may consider the Hurwitz chain associated to $\xi$ (see [4]), consisting of pairs $\left(\frac{p_{k}^{\prime}}{q_{k}^{\prime}}, \frac{p_{k}^{\prime \prime}}{q_{k}^{\prime \prime}}\right), k \in \mathrm{N}$, of elements in the Farey sequence $\mathcal{F}_{1}, \mathcal{F}_{2}, \ldots$, framing $\xi$ (a pair can give the frame for several consecutive Farey sequences). Therefore $\frac{p_{k}^{\prime}}{q_{k}^{\prime}}$ and $\frac{p_{k}^{\prime \prime}}{q_{k}^{\prime \prime}}$ are consecutive elements in some Farey sequence $\mathcal{F}_{n}$. But the first Farey sequence containing an element (necessarily unique, because two consecutive elements in a Farey sequence have different denominators) strictly between these two fractions is the one containing the mediant $\frac{p_{k}^{\prime}+p_{k}^{\prime \prime}}{q_{k}^{\prime}+q_{k}^{\prime \prime}}$ (cf. [2, Thm. 29]). We conclude that the next pair $\left(\frac{p_{k+1}^{\prime}}{q_{k+1}^{\prime}}, \frac{p_{k+1}^{\prime \prime}}{q_{k+1}^{\prime \prime}}\right)$ in the Hurwitz chain is either $\left(\frac{p_{k}^{\prime}}{q_{k}^{\prime}}, \frac{p_{k}^{\prime}+p_{k}^{\prime \prime}}{q_{k}^{\prime}+q_{k}^{\prime \prime}}\right)$ or $\left(\frac{p_{k}^{\prime}+p_{k}^{\prime \prime}}{q_{k}^{\prime}+q_{k}^{\prime \prime}}, \frac{p_{k}^{\prime \prime}}{q_{k}^{\prime \prime}}\right)$, according to whether the mediant $\frac{p_{k}^{\prime}+p_{k}^{\prime \prime}}{q_{k}^{\prime}+q_{k}^{\prime \prime}}$ is larger or smaller than $\xi$. This way of producing the Hurwitz chain is also called the Farey process ${ }^{5}$ in [7].

Example 2. The Hurwitz chain associated to $\frac{85}{38}=$ $2+\frac{9}{38}$ is

$$
\begin{aligned}
& \left(\frac{2}{1}, \frac{3}{1}\right) \quad\left(\frac{2}{1}, \frac{5}{2}\right) \quad\left(\frac{2}{1}, \frac{7}{3}\right) \quad\left(\frac{2}{1}, \frac{9}{4}\right) \quad\left(\frac{11}{5}, \frac{9}{4}\right) \\
& \left(\frac{20}{9}, \frac{9}{4}\right) \quad\left(\frac{29}{13}, \frac{9}{4}\right) \quad\left(\frac{38}{17}, \frac{9}{4}\right) \quad\left(\frac{38}{17}, \frac{47}{21}\right) \quad\left(\frac{85}{38}\right) \text {. }
\end{aligned}
$$

When $\xi$ is rational it coincides with one of the mediants $\frac{p_{k}^{\prime}+p_{k}^{\prime \prime}}{q_{k}^{\prime}+q_{k}^{\prime \prime}}$, for some $k$. Beyond this index $k$ one may extend the Hurwitz chain keeping either the pair $\left(\frac{p_{k}^{\prime}}{q_{k}^{\prime}}, \frac{p_{k}^{\prime}+p_{k}^{\prime \prime}}{q_{k}^{\prime}+q_{k}^{\prime \prime}}\right)$ or the pair $\left(\frac{p_{k}^{\prime}+p_{k}^{\prime \prime}}{q_{k}^{\prime}+q_{k}^{\prime \prime}}, \frac{p_{k}^{\prime \prime}}{q_{k}^{\prime \prime}}\right)$. We prefer not to consider here the Hurwitz chain of

${ }^{5}$ As an exercise, show that $\frac{p_{k}^{\prime}+p_{k}^{\prime \prime}}{q_{k}^{\prime}+q_{k}^{\prime \prime}}$ is on the same side of $\xi$ as $\frac{p_{k}^{\prime}}{q_{k}^{\prime}}$ (resp. $\left.\frac{p_{k}^{\prime \prime}}{q_{k}^{\prime \prime}}\right)$ if and only if $\left|q_{k}^{\prime} \xi-p_{k}^{\prime}\right|>\left|q_{k}^{\prime \prime} \xi-p_{k}^{\prime \prime}\right|$ (resp. $\left|q_{k}^{\prime} \xi-p_{k}^{\prime}\right|<\left|q_{k}^{\prime \prime} \xi-p_{k}^{\prime \prime}\right|$ ). Deduce that the sequence of best approximations of type 0 of $\xi$ alternates under and above $\xi$. rational numbers beyond the mediant it coincides with. Therefore, rational numbers are the numbers with a finite Hurwitz chain (the last pair reducing to the given rational).

Remark 1. When $\xi$ does not belong to the interval $[0,1]$, our definition of the Hurwitz chain differs from that described in [1]; see also [4]. In these references, supposing $\xi>0$, one starts from the pair $\left(\frac{0}{1}, \frac{1}{0}\right)$, and the Hurwitz chain begins with $\left(\frac{1}{1}, \frac{1}{0}\right)\left(\frac{2}{1}, \frac{1}{0}\right) \ldots\left(\frac{[\xi]}{1}, \frac{1}{0}\right)$ before the appearance of the pair $\left(\frac{[\xi]}{1}, \frac{[\xi]+1}{1}\right)$ of elements of $\mathcal{F}_{1}$ framing $\xi$.

The Hurwitz chain of $\xi$ produces both sequences of lower and upper rational approximations of $\xi$. One deduces also the Hurwitz sequence built from the components of the pairs in the Hurwitz chain, not repeated and ordered by increasing denominators (this is the sequence of mediants appearing successively in the Farey process). The Hurwitz sequence $\left(\frac{p_{k}}{q_{k}}\right)_{k \in \mathbf{N}}$ of $\xi$ starts with $\frac{p_{0}}{q_{0}}=$ $\frac{[\xi]}{1} \frac{p_{1}}{q_{1}}=\frac{[\xi]+1}{1} \frac{p_{2}}{q_{2}}=\frac{2[\xi]+1}{2} \ldots$. It contains all the "rational approximations" of $\xi$ (in any reasonable sense $)^{6}$ and in particular the best approximations of type 0 and 1 already discussed. However, one still has to learn how to sort these best approximations out of the complete Hurwitz sequence.

Example 3. The Hurwitz sequence of $\frac{85}{38}$ is $23 \frac{5}{2} \quad \frac{7}{3} \frac{9}{4} \quad \frac{11}{5} \quad \frac{20}{9} \quad \frac{29}{13} \quad \frac{38}{17} \quad \frac{47}{21} \quad \frac{85}{38}$, and the best approximations of type 0 are $2 \frac{9}{4} \quad \frac{38}{17} \frac{85}{38}$.

The Hurwitz sequence $\left(\frac{p_{k}}{q_{k}}\right)_{k \in \mathbf{N}}$ of $\xi$ is characterized by the sequence of signs encoding whether the corresponding approximation of $\xi$ is below or above $\xi$. More precisely, one reconstructs step by step the Hurwitz sequence of $\xi$ from the integer part of $\xi$ and its characteristic sequence of signs $\varepsilon_{k+1}:=\frac{p_{k}-q_{k} \xi}{\left|p_{k}-q_{k} \xi\right|}, k \geq 2$. We set $\varepsilon_{1}=-1$ and $\varepsilon_{2}=+1$ in order to complete the characteristic sequence in $\begin{array}{lllll}\varepsilon_{1} & \varepsilon_{2} & \varepsilon_{3}\end{array} \ldots$ (note the shift of index in comparison with the Hurwitz sequence, which starts with index 0 ). To recover the Hurwitz sequence from the characteristic sequence, one checks that the term $\frac{p_{k+1}}{q_{k+1}}$ is equal to the mediant of $\frac{p_{k}}{q_{k}}$ and $\frac{p_{\ell}}{q_{\ell}}$, where $\ell$ is the largest index $<k$ satisfying $\varepsilon_{k} \varepsilon_{\ell}=-1\left(\frac{p_{\ell}}{q_{\ell}}\right.$ is the surviving component in the pairs of the Hurwitz chain until the next change of sign), and that the corresponding pair in the Hurwitz chain is

\footnotetext{
${ }^{6}$ More precisely, a rational number $\frac{p}{q}$ is a (rational) approximation of $\xi$ if there is no other rational number of denominator less than or equal to $q$ between $\frac{p}{q}$ and $\xi$. Obviously, rational numbers that do not satisfy this property cannot qualify for the status of approximation. However, remember that, given an approximation, there may be other approximations closer to $\xi$ and of smaller denominators but on the other side of $\xi$.
} 
deduced from the signs $\varepsilon_{\ell}, \varepsilon_{k}$, and $\varepsilon_{k+1}$. Whenever $\xi$ is a rational number and $k$ is the first index such that $p_{k}-q_{k} \xi=0$, one may set by convention $\varepsilon_{k+1}=\varepsilon_{k}$, and the characteristic sequence stops with $k+1$ terms.

Example 4. The characteristic sequence of $\frac{85}{38}$ is $-++++-{ }_{-}-++$.

Remarkably, one can also recover straightforwardly the expansion of $\xi$ as a regular continued fraction from its characteristic sequence. Putting aside the first sign and, with our convention above, on the last sign in case $\xi$ is a rational number, one checks that the length of the blocks of identical signs in the characteristic sequence are the partial quotients in the expansion of $\xi$ as a regular continued fraction, except for the integer part of $\xi$, which starts equally the continued fraction and the Hurwitz sequence in our description. The approximations of type 0 , that is, the convergents (also known as approximants) of this regular continued fraction, are the elements $\frac{p_{k}}{q_{k}}$ of the Hurwitz sequence positioned just before a change of sign (i.e., such that $\varepsilon_{k+1} \varepsilon_{k+2}=-1$ ); see [4, §§3 \& 5]. ${ }^{7}$

The Hurwitz sequence of $\xi$ is represented on the Farey graph by both paths minimizing the surface that each of them cuts with the ray determined by $\xi$, but with the constraint that these paths never cross the ray. Therefore, there are two paths approaching the ray determined by $\xi$ from one or the other side. The radial edges of each of these paths enumerate the sequences of best approximations of $\xi$ from below and from above respectively. In Klein's interpretation, the vertices crossed by these two paths are all the vertices touched (corners and intermediate ones) by the two black strings in Figure 6.

\section{Continued Fraction Expansions}

A very good reference for this section is O. Perron [6]; we refer the reader to this monograph for most of the definitions and statements. We start with a quick tour of the zoo of continued fraction expansions of real numbers. We introduce the classical terminology for continued fractions, along with practical algorithms computing the complete quotients and partial fractions for several continued fraction expansions, selecting some remarkable sequences of approximations of a given real number.

\footnotetext{
${ }^{7}$ This is because the approximations of type 0 alternate under and above $\xi$; see footnote 5 and also the next section.
}

\section{The Zoo of Continued Fraction Expansions}

The "ordinary" expansion in continued fractions is well known; its convergents give the sequence of best approximations of type 0 of a real number. Continued fractions of the form

$$
a_{0}+\frac{1}{a_{1}+\frac{1}{a_{2}+\frac{1}{a_{3}+\ldots,}}}=\left[a_{0}, a_{1}, a_{2}, \ldots\right]
$$

with partial quotients $a_{1}, a_{2}, \ldots$ positive integers and $a_{0}$ an integer, are called regular. Infinite, regular continued fractions are convergent (that is, their sequences of convergents converge!) and their limits are in bijection with the set of all irrational real numbers. To each rational number correspond exactly two regular continued fractions which are finite. The numbers of their partial quotients differ by 1: the longest has 1 as last partial quotient and the shortest not. Expansions in regular continued fractions of quadratic numbers are those that are ultimately periodic (Lagrange's theorem). Purely periodic expansions correspond to reduced real quadratic numbers, that is, real quadratic numbers larger than 1 such that their conjugates lay between -1 and 0 (Galois's theorem).

Here is the well-known algorithm computing the expansion in regular continued fractions of the real number $\xi$ in the form (4), namely, $\xi=\left[a_{0}, a_{1}, a_{2}, \ldots\right]$ :

Algorithm $^{8}$ RCF:

-1) $v_{0}=\xi$

k) $a_{k}=\left[v_{k}\right]$, then $v_{k+1}:=\frac{1}{v_{k}-a_{k}}>1$;

the convergents of which are all the approximations of $\xi$ of type 0 ordered by strictly increasing denominators; see also [2, Chap. X] for example.

More generally, semi-regular continued fractions are those of the form ${ }^{9}$

(5)

$$
a_{0}+\frac{\varepsilon_{1}}{a_{1}+\frac{\varepsilon_{2}}{a_{2}+\frac{\varepsilon_{3}}{a_{3}+\ldots}}}=a_{0}+\underline{\varepsilon_{1} \sqrt{a_{1}}}+\underline{\varepsilon_{2}} \sqrt{a_{2}}+\ldots
$$

where $\varepsilon_{i} \in\{ \pm 1\}$ for $i \geq 1, a_{1}, a_{2}, \ldots$ are positive integers satisfying $a_{i}+\varepsilon_{i+1} \geq 1$ for $i \geq 1$ and, if the continued fraction is infinite, $a_{i}+\varepsilon_{i+1} \geq 2$ for an infinity of $i$; whereas if the continued fraction is finite, one requires that its last partial quotient be $>1$ (except when it coincides with the initial term). Semi-regular continued fractions are also

\footnotetext{
${ }^{8}$ This algorithm and the others described in the sequel stop as soon as $v_{k+1}=\infty$ (see the definition of this parameter in each case); otherwise they continue indefinitely. They work on the principle of a "whi 1e" loop that must be repeated from $k=0$ on indefinitely, unless one gets $v_{k+1}=\infty$ for some $k$.

${ }^{9}$ Observe that with the notation in (4) and (5) one has $\left[a_{0}, a_{1}, a_{2}, \ldots\right]=a_{0}+1 \sqrt{a_{1}}+1 \sqrt{a_{2}}+\ldots$
} 
convergent, and to each irrational real number $\xi$ and sequence of signs $\left(\varepsilon_{i}\right)_{i \in \mathbf{N}^{*}}$ corresponds an expansion in semi-regular continued fractions having $\varepsilon_{i}$ as partial numerators and $\xi$ as limit. A rational number of denominator $q$ has exactly $q$ expansions in semi-regular continued fractions. Periodic semi-regular continued fractions are in bijection with the expansions of quadratic real numbers for prescribed periodic sequences of signs. Here is an algorithm computing the semiregular expansion in continued fractions of a real number $\xi$ with a given prescribed sequence of signs $\varepsilon_{1}, \varepsilon_{2}, \ldots$ : Algorithm $^{10}$ SRCF:

-1) $v_{0}=\xi$;

k) $a_{k}=\left\{\begin{array}{ll}\left\lfloor v_{k}\right\rfloor & \text { if } \varepsilon_{k+1}=+1 \\ \left\lceil v_{k}\right\rceil \text { if } & \varepsilon_{k+1}=-1\end{array}\right.$, then $v_{k+1}:=$ $\frac{1}{\left|v_{k}-a_{k}\right|}>1$.

In general, the sequence of convergents of a semi-regular continued fraction is not produced with increasing denominators; some sparse partial fractions $-1 \sqrt{1}$ may alter this natural order.

Of course, prescribing the sequence of all positive signs, one recovers the expansion in regular continued fractions as one of the expansions in semi-regular continued fractions. On the opposite side, one may prescribe the sequence of all negative signs, possibly allowing a plus sign for the first one. The convergents of these continued fractions are all the approximations of the real numbers $\xi$ from above when $\varepsilon_{1}=-1$ and from below when $\varepsilon_{1}=+1$, ordered by strictly increasing denominators. ${ }^{11}$ Here are the algorithms specialized from SRCF computing these "negative" continued fractions:

Algorithm NCF:

-1) $v_{0}=\xi$

k) $a_{k}=\left\lceil v_{k}\right\rceil$, then $v_{k+1}:=\frac{1}{a_{k}-v_{k}}>1$ and $\varepsilon_{k+1}=-1$

the convergents of which are the approximations of $\xi$ from above.

Algorithm NCF+:

-1) $v_{0}=\xi$

0) $a_{0}=\left\lfloor v_{0}\right\rfloor$, then $v_{1}:=\frac{1}{v_{0}-a_{0}}>1$ and $\varepsilon_{1}=+1$

k) $a_{k}=\left\lceil v_{k}\right\rceil$, then $v_{k+1}:=\frac{1}{a_{k}-v_{k}}>1$ and $\varepsilon_{k+1}=-1$

the convergents of which are the approximations of $\xi$ from below.

\footnotetext{
${ }^{10}$ Here we denote by $\lceil\star\rceil$ the smallest integer larger than $\star$ and by $\lfloor\star\rfloor$ the usual integer part (also denoted by [*] elsewhere in this text, for example, in algorithm RCF).

${ }^{11}$ The reader can check that the daunting partial fraction -1/ $\sqrt{1}$ never occurs in these two "negative" continued fractions.
}

Continued fractions of the form (5) with $\varepsilon_{i} \in$ $\{ \pm 1\}$ and $a_{i}$ positive integers are called unitary. If such a continued fraction does not contain the partial fraction $-1 \sqrt{1}$ it converges. ${ }^{12} \mathrm{~J}$. Goldman $[1$, Thm. 6] shows that the convergents of a unitary continued fraction that does not contain any partial fraction equal to $-1 \sqrt{1}$ all belong to the Hurwitz sequence of its limit. In particular, the Hurwitz sequence of a real number $\xi$ identifies with the sequence of convergents of a unitary continued fraction (however, not semi-regular), which we call a complete continued fraction, such that $\underline{\varepsilon_{i}} \overline{a_{i}}=1 \sqrt{1}$ or $-1 \sqrt{2}$, for $i \geq 1$ and $a_{0}=[\xi]$. If the Hurwitz sequence is finite, the continued fraction is also finite; whereas if the Hurwitz sequence is infinite, then $1 \sqrt{1}$ appears infinitely many times in the continued fraction. Reciprocally, the sequence of convergents of a continued fraction of this type is the Hurwitz sequence of its limit; cf. [1]. Here is how to compute the complete continued fraction of the real number $\xi$ :

Algorithm CCF:

-1) $v_{0}=\xi$

0) $a_{0}=\left\lfloor v_{0}\right\rfloor$, then $v_{1}:=\frac{1}{v_{0}-a_{0}}>0$ and $\varepsilon_{1}=+1$;

k) $a_{k}=\left\{\begin{array}{lll}1 & \text { if } & \varepsilon_{k}=+1 \\ 2 & \text { if } & \varepsilon_{k}=-1\end{array}\right.$, then $v_{k+1}:=\frac{1}{\left|v_{k}-a_{k}\right|}$ and $\varepsilon_{k+1}$ is the sign of $v_{k}-a_{k}$;

the convergents of which form the complete Hurwitz sequence of $\xi$, ordered by strictly increasing denominators.

On another hand, given a sequence of rational numbers $\left(\frac{p_{k}}{q_{k}}\right)_{k \in \mathrm{N}}$ ordered by strictly increasing denominators, this sequence can be obtained as the sequence of convergents of a unitary continued fraction (with no partial fraction $-1 \sqrt{1}$ ) if and only if any two successive terms satisfy $\left|p_{k+1} q_{k}-p_{k} q_{k+1}\right|=1$.

A semi-regular continued fraction is said to be singular if one has $a_{i} \geq 2$ and $a_{i}+\varepsilon_{i} \geq 2$ for all $i \in \mathbf{N}^{*}$. Every real number has an expansion in singular continued fractions, unique if it is not equivalent to $\frac{\sqrt{5}-1}{2}$ (under the action of $\mathrm{Sl}_{2}(\mathbf{Z})$ ). Again, the expansions of real quadratic numbers in singular continued fractions are those which are periodic; cf. [3, §4]. The following algorithm computes the expansion of the real number $\xi$ in a singular continued fraction.

\section{Algorithm SGCF:}

$$
\text { -1) } v_{0}=\xi
$$

\footnotetext{
${ }^{12}$ Actually, it suffices that the occurrences of the partial fraction $-1 \sqrt{1}$ are sparse enough (certainly no two consecutive), as in the case of semi-regular continued fractions already mentioned.
} 
k) $a_{k}=\left\lfloor v_{k}+\frac{3-\sqrt{5}}{2}\right\rfloor$, then $v_{k+1}:=\frac{1}{\left|v_{k}-a_{k}\right|} \geq$ $\frac{\sqrt{5}+1}{2}$ and $\varepsilon_{k+1}$ is the sign of $v_{k}-a_{k}$;

the convergents of which, $\frac{p}{q}$, satisfy $\left|\xi-\frac{p}{q}\right| \leq$ $\frac{\sqrt{5}-1}{2 q^{2}}$ and are ordered by strictly increasing denominators.

Note that in algorithm $S G C F$ the partial quotient $a_{k}$ is the integer closest to $v_{k}+1-\frac{\sqrt{5}}{2}$, with the choice of the larger one if $v_{k}-\frac{\sqrt{5}}{2}$ is a half integer.

One may also expand a real number in a continued fraction along the lines of the usual algorithm $R C F$, but selecting the closest integer (rather than taking the integer part) at each step. One then obtains a semi-regular continued fraction satisfying, furthermore, $a_{i} \geq 2$ and $a_{i}+\varepsilon_{i+1} \geq 2$ for all $i \in \mathbf{N}^{*}$. For rational numbers this expansion is the shortest, while for an irrational, quadratic real number it is periodic; cf. [3, §2]. Here is the corresponding algorithm that computes the closest integer continued fraction expansion of $\xi$ :

Algorithm CICF:

-1) $v_{0}=\xi$

k) $a_{k}=\left\lceil v_{k}-\frac{1}{2}\right\rceil$, then $v_{k+1}:=\frac{1}{\left|v_{k}-a_{k}\right|} \geq 2$ and $\varepsilon_{k+1}$ is the sign of $v_{k}-a_{k}$;

the convergents of which, $\frac{p}{q}$, satisfy $\left|\xi-\frac{p}{q}\right| \leq \frac{\sqrt{5}-1}{2 q^{2}}$ and are ordered by strictly increasing denominators.

In algorithm CICF the partial quotient $a_{k}$ is the integer closest to $v_{k}$, with the choice of the smallest one when $v_{k}$ is a half integer. ${ }^{13}$

More generally, McKinney [5] has studied the expansions in continued fractions along the closest integer after shifting by a fixed real number $\lambda$, which he calls $\lambda$-development.

Any convergent $\frac{p}{q}$ of the expansions in singular continued fractions and along the closest integer continued fraction of a real number are best approximations of type 0 satisfying

$$
\left|\xi-\frac{p}{q}\right| \leq \frac{\sqrt{5}-1}{2 q^{2}} .
$$

The lists of convergents of these two continued fractions overlap, but also contain distinct approximations. However, the union of the two lists does not contain all the best approximations of type 0 satisfying (6). Furthermore, the rational numbers satisfying (6) are not necessarily best approximations of type 0 or even 1 .

It is trickier to devise an algorithm selecting precisely all the rational approximations of $\xi$ satisfying (6), ordered by strictly increasing

${ }^{13}$ With this choice the continued fraction ends as $\left\lfloor v_{k}\right\rfloor+1 \sqrt{2}$ when $v_{k}$ is a half integer for some $k$ (note that $\xi$ is then a rational number). With the other choice the continued fraction would end as $\left\lceil v_{k}\right\rceil+-1 \sqrt{2}$, but the penultimate convergent may not be an approximation of type 0 . denominators. First, one has to verify that the sequence $\left(\frac{p_{k}}{q_{k}}\right)_{k \in \mathrm{N}}$ of these approximations satisfies $\left|p_{k+1} q_{k}-p_{k} q_{k+1}\right|=1$ for all $k \in \mathbf{N}$, which is true but not obvious. In fact, for any real number $\varpi \in\left[\frac{1}{2}, \frac{2}{3}\right]$ the sequence of rational approximations of $\xi$ satisfying

$$
\left|\xi-\frac{p_{k}}{q_{k}}\right| \leq \frac{\varpi}{q_{k}^{2}}
$$

also satisfies $\left|p_{k+1} q_{k}-p_{k} q_{k+1}\right|=1$ and can therefore be obtained as the sequence of convergents of a unitary continued fraction. This may no longer be true for a positive number $\varpi$ strictly smaller than $\frac{1}{2}$ or strictly larger than $\frac{2}{3}$.

For $\varpi \in\left[\frac{1}{2}, \frac{2}{3}\right]$ the following algorithm $D C F(\varpi)$ produces the continued fraction that has the sequence of all rational approximations of $\xi$ satisfying (7) as sequence of convergents. It does not seem to appear in the literature. Thus, checking that it indeed selects the asserted sequence of approximations is a real challenge proposed to the reader.

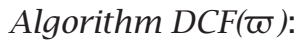

$$
\begin{aligned}
& \text {-1) } v_{0}=\xi ; \text { 0) } a_{0}=\left\{\begin{array}{l}
\left\lfloor v_{0}\right\rfloor \text { if } v_{0}-\left\lfloor v_{0}\right\rfloor \leq \varpi \\
\left\lceil v_{0}\right\rceil \text { otherwise }
\end{array}\right. \\
& v_{1}:=\frac{1}{\left|v_{0}-a_{0}\right|}, \\
& \varepsilon_{1} \text { is the sign of } v_{0}-a_{0}, r_{1}:=0 \\
& \text { and } e_{1}:=\left|v_{0}-a_{0}\right| ; \\
& \text { k) } a_{k}=\left\{\begin{array}{l}
\frac{3-\varepsilon_{k}}{2} \text { if } e_{k}\left|v_{k}-\frac{3-\varepsilon_{k}}{2}\right|\left(\frac{3-\varepsilon_{k}}{2}+\varepsilon_{k} r_{k}\right) \leq \varpi \\
\left|v_{k}-\frac{1}{2 e_{k}}\left(1-\sqrt{1-4 \varpi e_{k}}\right)\right| \text { otherwise }
\end{array}\right. \\
& v_{k+1}:=\frac{1}{\left|v_{k}-a_{k}\right|}, \varepsilon_{k+1} \text { is the sign of } \\
& v_{k}-a_{k}, r_{k+1}:=\frac{1}{a_{k}+\varepsilon_{k} r_{k}} \text { and } e_{k+1}:= \\
& \frac{e_{k}}{v_{k+1} r_{k+1}} ;
\end{aligned}
$$

the convergents of which, $\frac{p}{q}$, are all the approximations of $\xi$ satisfying $\left|\xi-\frac{p}{q}\right| \leq \frac{\varpi}{q^{2}}$, ordered by strictly increasing denominators.

The reader may like to check that the quantities $e_{k}$ introduced in the algorithm $D C F(\varpi)$ satisfy $e_{k}\left(v_{k}+\varepsilon_{k} r_{k}\right)=1$. Thus, eliminating $e_{k}$, the definition of $a_{k}$ in the second case of the general step can be rewritten accordingly as

$$
\begin{aligned}
& a_{k}= \\
& \left\lceil\frac{1}{2}\left(v_{k}-\varepsilon_{k} r_{k}+\sqrt{\left(v_{k}+\varepsilon_{k} r_{k}\right)^{2}-4 \varpi\left(v_{k}+\varepsilon_{k} r_{k}\right)}\right)\right]
\end{aligned}
$$

and the condition in the first case reads

$$
\left|\nu_{k}-\frac{3-\varepsilon_{k}}{2}\right|\left(\frac{3-\varepsilon_{k}}{2}+\varepsilon_{k} r_{k}\right) \leq \varpi\left(\nu_{k}+\varepsilon_{k} r_{k}\right) .
$$

Furthermore, if the condition in the first case is not satisfied, then $v_{k}+\varepsilon_{k} r_{k} \geq 4 \varpi$ and the square root in the definition of $a_{k}$ in the second case is a definite nonnegative real number. 
For $\varpi=\frac{1}{2}$ one obtains the so-called diagonal continued fraction, which is semi-regular. It can also be computed by singularization ${ }^{14}$ of the regular continued fraction of the real number $\xi$. Its convergents $\frac{p}{q}$ are all the best approximations of $\xi$ of type 0 satisfying

$$
\left|\xi-\frac{p}{q}\right| \leq \frac{1}{2 q^{2}},
$$

since it is well known that rational numbers satisfying (8) are best approximations of $\xi$ of type 0 (cf. [2, Thm. 184]), and from any two consecutive best approximations of type 0 , at least one satisfies (8) (cf. [2, Thm. 183]). The expansions in diagonal continued fraction of quadratic numbers are periodic; see [6].

Finally, we want to introduce a last algorithm, the convergents of which form the remarkable sequence of best approximations of type 1 of a given real number $\xi$, ordered by increasing denominators. The corresponding continued fraction is unitary of the form (5). Here is this algorithm. ${ }^{15}$ As before, if $\xi$ is a rational number, we stop the algorithm as soon as $a_{k}=v_{k}$, that is, $v_{k+1}=\infty$.

Algorithm BACF:

-1) $v_{0}=\xi$

0) $a_{0}$ is the integer closest to $v_{0}$, let $v_{1}:=\frac{1}{\left|v_{0}-a_{0}\right|} \geq 2, \varepsilon_{1}$ the sign of $v_{0}-a_{0}$ and $r_{1}:=0$;

k) $a_{k}:=1+\left\lfloor\frac{1}{2}\left(v_{k}-\varepsilon_{k} r_{k}\right)\right\rfloor$, then $v_{k+1}:=$ $\frac{1}{\left|v_{k}-a_{k}\right|}>0, \varepsilon_{k+1}$ the sign of $v_{k}-a_{k}$ and $r_{k+1}:=\frac{1}{a_{k}+\varepsilon_{k} r_{k}}$;

the convergents of which are all the best approximations of $\xi$ of type 1 , ordered by strictly increasing denominators.

This expansion in unitary continued fractions of the real number $\xi$ is ultimately periodic if and only if $\xi$ is a quadratic number. ${ }^{16}$

Note that the quantity $r_{k+1}$ introduced above is just the ratio of the denominators of the $(k-1)$-th and $k$-th convergents of the continued fractions $a_{0}+\underline{\varepsilon_{1}} \sqrt{a_{1}}+\underline{\varepsilon_{2}} \sqrt{a_{2}}+\ldots$; that is, $r_{k+1}=$ $\frac{q_{k-1}}{q_{k}}=1 \sqrt{a_{k}}+\underline{\varepsilon_{k}} \sqrt{a_{k-1}}+\cdots+\underline{\varepsilon_{2}} \sqrt{a_{1}}$. But, although this parameter is a rational number, it does not

${ }^{14}$ The singularization of a continued fraction $a_{0}+\varepsilon_{1} \sqrt{a_{1}}+$ $\cdots+\varepsilon_{k+1} \sqrt{a_{k+1}}+\ldots$ at $a_{k+1}$ is the continued fraction $a_{0}+\underline{\varepsilon_{1}} \sqrt{a_{1}}+\cdots+\underline{\varepsilon_{k-1}} \sqrt{a_{k-1}}+\underline{\varepsilon_{k}} \sqrt{a_{k}+1}+\underline{-1} \sqrt{a_{k+2}+1}+$ $\varepsilon_{k+3} \overline{a_{k+3}}+\ldots$ Here, one has to singularize all the partial quotients $a_{k+1}$ of the regular continued fraction such that the convergent $\frac{p_{k}}{q_{k}}$ satisfies $\left|\xi-\frac{p_{k}}{q_{k}}\right|>\frac{1}{2 q_{k}^{2}}$.

${ }^{15}$ Computations establishing the previous statement can be found in: P. Philippon, Un oil et Farey, http://ha1 . archives-ouvertes . fr/ha1-00488471 (02/06/2010).

${ }^{16}$ See the reference in footnote 15 for details of the proof of this fact. need to be computed exactly. It suffices to compute it as a real number with the same precision as the parameter $v_{k}$.

\section{Sequences of Approximations}

We explain in this subsection how the complete continued fraction expansion (algorithm CCF in the previous subsection) of a real number $\xi$ is simply related to its expansion in regular continued fractions (algorithm RCF in the previous subsection).

Recall that the sequence of convergents of a continued fraction of the form (5) is given by $\left\{\begin{array}{l}p_{0}=a_{0} \\ q_{0}=1\end{array}\right.$ and, with the convention $\left\{\begin{array}{l}p_{-1}=1 \\ q_{-1}=0\end{array}\right.$,

$$
\left\{\begin{array}{l}
p_{k+1}=a_{k+1} p_{k}+\varepsilon_{k+1} p_{k-1} \\
q_{k+1}=a_{k+1} q_{k}+\varepsilon_{k+1} q_{k-1}
\end{array} \quad \text { for } k \in \mathbf{N} .\right.
$$

Approximations of type 0 are the convergents of the expansion in regular continued fractions of a real number $\xi$. On another hand, the Hurwitz sequence of $\xi$ is made of all the convergents $\left(\frac{p_{k}}{q_{k}}\right)_{k \in \mathbf{N}}$ of this expansion in regular continued fractions plus all the intermediate convergents (of denominators between $q_{k}$ and $q_{k+1}$ ):

$$
\frac{\lambda p_{k}+p_{k-1}}{\lambda q_{k}+q_{k-1}}, \quad \lambda=1, \ldots, a_{k+1}-1, \quad k \in \mathbf{N}^{*} .
$$

The complete expansion in unitary continued fractions of a real number $\xi$ is related in a simple way to the expansion in regular continued fractions. If $\xi=\left[c_{0}, c_{1}, c_{2}, \ldots\right]$ is the sequence of partial quotients of an irrational real number $\xi$, the complete expansion in unitary continued fractions is written

$$
\begin{aligned}
& \xi=[\xi]+1[1]+[\underbrace{1 \sqrt{1}+\underline{-1 \sqrt{2}+\cdots+\underline{-1} 2}}]_{i \geq 1} \\
& c_{i} \text { term(s) }
\end{aligned}
$$

where the overline means that each time the motif between brackets is reproduced, the integral index $i$ must be incremented by 1 starting from 1 up to $+\infty$. Each successive group contains $c_{i}$ terms, the first one being $1 \sqrt{1}$ and the possible followers being $-1\left[2\right.$. When $\xi=\left[c_{0}, c_{1}, \ldots, c_{m}\right]$ is a rational number there are $m$ groups, and the last one contains only $c_{m}-1$ terms so that the total length of the expansion is $c_{1}+\cdots+c_{m}$ (leaving aside the initial integer part). For all integer $i$ we set $\tilde{c}_{i}=c_{1}+\cdots+c_{i}$. One has the following correspondence between the complete continued fraction expansion, Hurwitz sequence, and the characteristic sequence of $\xi$ :

$$
\begin{aligned}
& {[\xi]+1 \sqrt{1}+[\quad 1 \sqrt{1}+\underline{-1 \sqrt{2}}+\ldots+\underline{-1} \sqrt{2}+\underline{-1} \sqrt{2}]_{i \geq 1}} \\
& \begin{array}{llllll}
p_{0} & \frac{p_{1}}{q_{1}}
\end{array}\left[\begin{array}{lllll}
\frac{p_{\tilde{c}_{i-1}+2}}{q_{\tilde{c}_{i-1}+2}} & \frac{p_{\tilde{c}_{i-1}+3}}{q_{\tilde{c}_{i-1}+3}} & \ldots & \frac{p_{\tilde{c}_{i}}}{q_{\tilde{c}_{i}}} & \frac{p_{\tilde{c}_{i}+1}}{q_{\tilde{c}_{i}}+1}
\end{array}\right]_{i \geq 1} \\
& +\left[\begin{array}{lllll}
(-1)^{i-1} & (-1)^{i-1} & \ldots & (-1)^{i-1} & (-1)^{i}
\end{array}\right]_{i \geq 1} .
\end{aligned}
$$

Beware that, in the above picture, when $c_{i}=1$ or $c_{i}=2$ the partial fractions corresponding to 
the convergents $\frac{p_{\tilde{c}_{i}}}{q_{\tilde{c}_{i}}}$ and $\frac{p_{\tilde{c}_{i}+1}}{q_{\tilde{c}_{i}+1}}$ are not necessarily $-1 \sqrt{2}$, because the first term of each group between brackets is always 1$] 1$ and the fraction $\frac{p_{\tilde{c}_{i}}}{q_{\tilde{c}_{i}}}$ may even stand before the beginning of that group when $c_{i}=1$. The convergents of the expansion in regular continued fractions (i.e., the best approximations of type 0) are $\frac{p_{0}}{q_{0}}$ and $\frac{p_{\tilde{c}_{i}}}{q_{\tilde{c}_{i}}}, i \geq 1$.

\section{Complete Quotients}

The quantities $v_{k}$ introduced in the descriptions of the algorithms in "The zoo of continued fraction expansions" are related to the complete quotients of the continued fraction of the form (5); that is, for $k \in \mathbf{N}$,

$$
v_{k}=a_{k}+\frac{\varepsilon_{k+1}}{a_{k+1}+\frac{\varepsilon_{k+2}}{a_{k+2}+\frac{\varepsilon_{k+3}}{a_{k+3}+\ldots}}}
$$

Supposing that the fraction (5) converges towards a real number $\xi$ and denoting by $v_{k}$ the $k$-th complete quotient as written above and by $\frac{p_{k}}{q_{k}}$ the $k$-th convergents, one then checks:

$$
\begin{aligned}
& \xi=\frac{v_{k+1} p_{k}+\varepsilon_{k+1} p_{k-1}}{v_{k+1} q_{k}+\varepsilon_{k+1} q_{k-1}}, \\
& v_{k+1}=-\varepsilon_{k+1} \frac{q_{k-1} \xi-p_{k-1}}{q_{k} \xi-p_{k}}, \\
& v_{k+1}=\frac{\varepsilon_{k+1}}{v_{k}-a_{k}}
\end{aligned}
$$

and

$v_{k+1}=-\underline{\varepsilon_{k+1} \sqrt{a_{k}}}+\underline{\varepsilon_{k}} \sqrt{a_{k-1}}+\cdots+\underline{\varepsilon_{2} \sqrt{a_{1}}}+\underline{\varepsilon_{1}} \sqrt{a_{0}-\xi}$.

The complete quotients of a regular continued fraction satisfy $v_{k}>1$ for any $k \in \mathbf{N}$. Therefore one can isolate the best approximations of type 0 among the convergents of the expansion in continued fractions along the closest integer or the complete expansion in unitary continued fractions, for example (or of any other expansion the sequence of convergents of which contains the sequence of best approximations of type 0 ordered by increasing denominators). Practically, one considers for these fractions the first product, $v_{2} \ldots v_{k_{1}+1}$ of modulus $>1$, then the following products, $v_{k_{i-1}+2} \ldots v_{k_{i}+1}$, again of modulus $>1$, which give (with $k_{0}=0$ ) the indices $k_{i}$ corresponding to the best approximations of type 0 . Then, the complete and partial quotients of the expansion in regular continued fractions are $u_{i+1}:=\left|v_{k_{i-1}+2} \ldots v_{k_{i}+1}\right|=\left|\frac{q_{k_{i-1}} \xi-p_{k_{i-1}}}{q_{k_{i}} \xi-p_{k_{i}}}\right|$ and $a_{i+1}:=\left[u_{i+1}\right]$.

Remark 2. If $\xi$ is the limit of the continued fraction (5), its complete quotients are given by the action of the following elements of $\mathrm{Gl}_{2}(\mathbf{Z})$ on $\xi$ $(k \in \mathrm{N})$ :

$$
\begin{aligned}
& v_{k+1} \\
& =\left[\varepsilon_{k+1}\right] S T^{-a_{k}}\left[\varepsilon_{k}\right] S T^{-a_{k-1}}\left[\varepsilon_{k-1}\right] S T^{-a_{k-2}} \\
& \ldots\left[\varepsilon_{1}\right] S T^{-a_{0}}(\xi) \text {, }
\end{aligned}
$$

where $S=x \mapsto \frac{1}{x}, T: x \mapsto X+1$, and $[\varepsilon]: x \mapsto \varepsilon x$.

\section{Intermediate Convergents}

For the best approximations of type 1, the usual way to look for them is to sort them from the intermediate convergents of the expansion in regular continued fractions. More precisely, if $\left(\frac{\tilde{p}_{i}}{\tilde{q}_{i}}\right)_{i \in \mathrm{N}}$ is the sequence of best approximations of type 0 of a real number $\xi=\left[c_{0}, c_{1}, c_{2} \ldots\right]$, the intermediate convergents between $\frac{\tilde{p}_{i-1}}{\tilde{q}_{i-1}}$ and $\frac{\tilde{p}_{i}}{\tilde{q}_{i}}$ are written

$$
\frac{\lambda \tilde{p}_{i}+\tilde{p}_{i-1}}{\lambda \tilde{q}_{i}+\tilde{q}_{i-1}}, \quad \lambda=1, \ldots, c_{i+1}-1 .
$$

Using the notation introduced in "Sequences of approximations", these intermediate convergents (9) are the elements of the Hurwitz sequence lying between $\frac{\tilde{p}_{i}}{\tilde{q}_{i}}=\frac{p_{\tilde{c}_{i}}}{q_{\tilde{c}_{i}}}$ and $\frac{\tilde{p}_{i+1}}{\tilde{q}_{i+1}}=\frac{p_{\tilde{c}_{i+1}}}{q_{\tilde{c}_{i+1}}}$ (recall that $\left.\tilde{c}_{i}=c_{1}+\cdots+c_{i}\right)$. Indeed we check

$$
\begin{aligned}
\left(\begin{array}{l}
p_{\tilde{c}_{i}+\lambda} \\
\tilde{q}_{c_{i}+\lambda}
\end{array}\right) & =\lambda\left(\begin{array}{c}
p_{\tilde{c}_{i}} \\
q_{\tilde{c}_{i}}
\end{array}\right)+\left(\begin{array}{c}
p_{\tilde{c}_{i-1}} \\
q_{\tilde{c}_{i-1}}
\end{array}\right) \\
& =\lambda\left(\begin{array}{c}
\tilde{p}_{i} \\
\tilde{q}_{i}
\end{array}\right)+\left(\begin{array}{c}
\tilde{p}_{i-1} \\
\tilde{q}_{i-1}
\end{array}\right) .
\end{aligned}
$$

Then, the best approximations of type 1 (that are not of type 0 ) read, for $\lambda, i \in \mathrm{N}^{*}$ :

$$
\frac{\lambda \tilde{p}_{i}+\tilde{p}_{i-1}}{\lambda \tilde{q}_{i}+\tilde{q}_{i-1}}
$$

with $c_{i+1}<2 \lambda \leq 2 c_{i+1}-2$ or $2 \lambda=c_{i+1}$ and $\left[c_{i+1}, c_{i}, \ldots, c_{2}, c_{1}\right]>\left[c_{i+1}, c_{i+2}, c_{i+3}, \ldots\right]$; see $[6$, Satz 22, p. 60]. Indeed, the condition to be satisfied for a best approximation of type 1 is $\left|\xi-\frac{\lambda \tilde{p}_{i}+\tilde{p}_{i-1}}{\lambda \tilde{q}_{i}+\tilde{q}_{i-1}}\right|<\left|\xi-\frac{\tilde{p}_{i}}{\tilde{q}_{i}}\right|$, which is equivalent to $2 \lambda>u_{i+1}-r_{i+1}$, where $u_{i+1}:=-\frac{\tilde{q}_{i-1} \xi-\tilde{p}_{i-1}}{\tilde{q}_{i} \xi-\tilde{p}_{i}}$ and $r_{i+1}:=\frac{\tilde{q}_{i-1}}{\tilde{q}_{i}}$. Since $c_{i+1}=\left[u_{i+1}\right]$ this gives $2 \lambda \geq c_{i+1}$ with equality if and only if $u_{i+1}-$ $\left[u_{i+1}\right]<r_{i+1}$, which is the condition stated because $u_{i+1}=\left[c_{i+1}, c_{i+2}, \ldots\right]$ and $\left[u_{i+1}\right]+r_{i+1}=$ $\left[c_{i+1}, c_{i}, \ldots, c_{2}, c_{1}\right]$.

Compare this approach with the algorithm BACF presented at the end of "The zoo of continued fraction expansions".

\section{Some Expansion in Continued Fractions, the Convergents of Which Are Best Approxima- tions of Types 0 and 1}

The algorithms described above are efficient and easy to program (with PARI, for example). We give here the expansion in continued fractions of type 
0 (ordinary) and of type 1 for some particular numbers. An overline indicates a sequence that must be repeated indefinitely with the possible increment of the parameter shown in subscript. Small points indicate that the expansion continues without any apparent motif. Finally, we describe precisely the structure of the expansion in continued fractions of best approximations of type 1 .

Remark 3. We have marked in red the partial fractions corresponding to convergents giving the best approximations of type 0 .

$$
\begin{aligned}
& \frac{1}{4}=[0,4]=0+\underline{1} \sqrt{3}+1 \sqrt{1} \\
& \frac{85}{38}=[2,4,4,2] \\
& =2+\underline{1} \sqrt{3}+\underline{1} \sqrt{1}+\underline{-1} \sqrt{4}+\underline{1 \sqrt{1}}+\underline{-1} \sqrt{2}+\underline{1} \sqrt{1} \\
& \frac{92}{13}=[7,13] \\
& =7+\underline{1} \sqrt{7}+1 \sqrt{1}+\underline{-1} \sqrt{2}+\underline{-1} \sqrt{2}+\underline{-1} \sqrt{2}+\underline{-1} \sqrt{2}+-1 \sqrt{2} \\
& \sqrt{2}=[1, \overline{2}]=1+\underline{1} \sqrt{2}+[\overline{1 \sqrt{1}+1 \sqrt{1}+\underline{-1 \sqrt{3}}}] \\
& \sqrt{3}=[1, \overline{1,2}]=2+\underline{-1 \sqrt{2}}+[\overline{1 \sqrt{1}+-1 \sqrt{2}+\underline{1} \sqrt{1}}] \\
& \sqrt{5}=[2, \overline{4}] \\
& =2+\underline{1} \sqrt{3}+[\overline{1 \sqrt{1}+\underline{-1} \sqrt{3}+1 \sqrt{1}+\underline{-1 \sqrt{2}}+\underline{-1 \sqrt{4}}]} \\
& \sqrt{6}=[2, \overline{2,4}]=2+1 \sqrt{2}+[\overline{1 \sqrt{2}+1 \sqrt{1}+-1 \sqrt{2}+-1 \sqrt{3}}] \\
& \sqrt{7}=[2, \overline{1,1,1,4}] \\
& =3+\underline{-1 \sqrt{2}}+[\overline{1 \sqrt{1}+\underline{1} \sqrt{2}+1 \sqrt{1}+\underline{-1 \sqrt{2}+-1 \sqrt{2}+1 \sqrt{1}}}] \\
& \frac{1+\sqrt{5}}{2}=[\overline{1}]=2+-1 \sqrt{2}+[\overline{1 \sqrt{1}}] \\
& \mathrm{e}=[2, \overline{1,2 n, 1}]_{n \in \mathbf{N}^{*}}=3+\underline{-1} \sqrt{2}+1 \sqrt{1}+\underline{-1} \sqrt{2} \\
& +[\overline{1 \sqrt{1}+1 \sqrt{n}+1 \sqrt{1}+\underbrace{-1 \sqrt{2}+\cdots+-1 \sqrt{2}+-1 \sqrt{2}}_{n}}]_{n \geq 2} \\
& \sqrt[q]{\mathrm{e}}=[\overline{1, q-1+2 q n, 1}]_{n \in \mathbf{N}} \\
& =1+1 \sqrt{\left[\frac{q+1}{2}\right]}+1 \sqrt{1}+\underbrace{-1 \sqrt{2}+\cdots+-1 \sqrt{2}+-1 \sqrt{2}}_{\left[\frac{q-2}{2}\right]}
\end{aligned}
$$

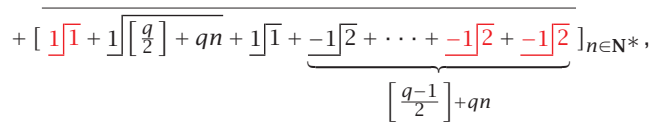

$$
\begin{aligned}
& \sqrt{\mathrm{e}}=[\overline{1,1+4 n, 1}]_{n \in \mathrm{N}}=2+\underline{-1} \sqrt{2} \\
& +[\overline{1 \sqrt{1}+1 \sqrt{1+2 n}+1 \sqrt{1}+\underbrace{-1 \sqrt{2}+\cdots+-1 \sqrt{2}+-1 \sqrt{2}}_{0+2 n}}]_{n \in \mathbf{N}^{*}} \\
& \sqrt[3]{\mathrm{e}}=[\overline{1,2+6 n, 1}]_{n \in \mathbf{N}}=1+1 \sqrt{2}+\underline{1} \sqrt{1}
\end{aligned}
$$

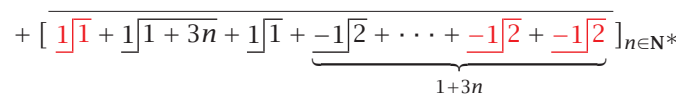

$$
\begin{aligned}
& \sqrt[4]{\mathrm{e}}=[\overline{1,3+8 n, 1}]_{n \in \mathbf{N}}=1+1 \sqrt{2}+1 \sqrt{1}+\underline{-1} \sqrt{2}
\end{aligned}
$$

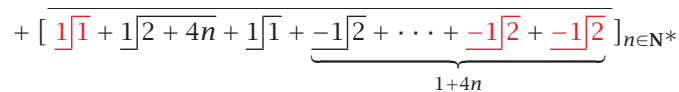

$$
\begin{aligned}
& \sqrt[5]{\mathrm{e}}=[\overline{1,4+10 n, 1}]_{n \in \mathrm{N}}=1+\underline{1} \sqrt{3}+\underline{1} \sqrt{1}+\underline{-1} \sqrt{2} \\
& +[\overline{1 \sqrt{1}+1 \sqrt{2+5 n}+1 \sqrt{1}+\underbrace{-1 \sqrt{2}+\cdots+-1 \sqrt{2}+-1 \sqrt{2}}_{2+5 n}}]_{n \in \mathbf{N}} * \\
& \pi=[3,7,15,1, \ldots] \\
& =3+1 \sqrt{4}+1 \sqrt{1}+-1 \sqrt{2}+-1 \sqrt{2}+-1 \sqrt{9}+1 \sqrt{1}+-1 \sqrt{2}+-1 \sqrt{2}+-1 \sqrt{2}+-1 \sqrt{2} \\
& +-1 \sqrt{2}+-1 \sqrt{2}+-1 \sqrt{2}+1 \sqrt{146}+1 \sqrt{1}+-1 \sqrt{2}+-1 \sqrt{2}+\ldots
\end{aligned}
$$

The first formulas, giving the expansions in regular continued fractions, are known; see [6] for example. Proving the equalities with the expansions in unitary continued fractions giving the best approximations of type 1 can be done as follows. One converts the latter expansion in the former with the help of the transformation (elimination of superfluous convergents, here $\frac{p_{i}}{q_{i}}$ ) taking the expansion:

$$
\cdots+\underline{c_{i}} \sqrt{a_{i}}+\underline{c_{i+1}} \sqrt{a_{i+1}}+\underline{c_{i+2}} \sqrt{a_{i+2}}+\underline{c_{i+3}} \sqrt{a_{i+3}}+\ldots
$$

to the transformed one:

$$
\begin{aligned}
\ldots & +\underline{a_{i+1} c_{i}} \sqrt{a_{i} a_{i+1}+c_{i+1}} \\
& +\frac{-c_{i+1} c_{i+2} \sqrt{a_{i+1} a_{i+2}+c_{i+2}}}{a_{i+1} c_{i+3} \sqrt{a_{i+3}}+\ldots}
\end{aligned}
$$

Furthermore, when the first approximation does not coincide with the integer part of the common limit number, we shorten the regular continued fraction from its initial term with the transformation:

$$
\begin{aligned}
a_{0}+ & 1 \sqrt{1}+\underline{c_{2}} \sqrt{a_{2}}+\ldots \\
& =\left(a_{0}+1\right)+\underline{-c_{2}} \sqrt{a_{2}+c_{2}}+\ldots .
\end{aligned}
$$

We then observe that the expansions deduced with these transformations coincide.

Combined with the results on intermediate convergents recalled in "Intermediate convergents", this method allows us to deduce the expansion in continued fractions of best approximations of type 1 from the expansion in regular continued fractions. More precisely, if $\xi=\left[c_{0}, c_{1}, c_{2}, \ldots\right]$ is the sequence of partial quotients of the expansion in regular continued fractions, we first set

$$
\begin{aligned}
& c_{0}^{\prime}:=\left\{\begin{array}{ll}
1 & \text { if } \xi>[\xi]+\frac{1}{2} \\
0 & \text { if } \xi<[\xi]+\frac{1}{2}
\end{array}\right. \text {, } \\
& c_{i}^{\prime}:=\left\{\begin{array}{ll}
c_{i+1} & \text { if } \xi>[\xi]+\frac{1}{2} \\
c_{i} & \text { if } \xi<[\xi]+\frac{1}{2}
\end{array} \quad \text { for } i \geq 1,\right.
\end{aligned}
$$

then $\lambda_{0}=0$ and for $i \geq 1$

$$
\lambda_{i}:= \begin{cases}\frac{c_{i}^{\prime}}{2} & \text { if } c_{i}^{\prime} \text { is even and } \\ & {\left[c_{i}^{\prime}, c_{i-1}^{\prime}, \ldots, c_{2}, c_{1}\right]} \\ & >\left[c_{i}^{\prime}, c_{i+1}^{\prime}, c_{i+2}^{\prime}, \ldots\right] \\ {\left[\frac{c_{i}^{\prime}}{2}\right]+1} & \text { otherwise }\end{cases}
$$


and finally

$$
\begin{aligned}
& a_{0}:=\left\{\begin{array}{ll}
{[\xi]+1} & \text { if } \xi>[\xi]+\frac{1}{2} \\
{[\xi]} & \text { if } \xi<[\xi]+\frac{1}{2}
\end{array}\right. \text { and } \\
& \underline{\varepsilon_{i} \sqrt{a_{i}}:}:=\left\{\begin{array}{ll}
\frac{-1 \sqrt{\lambda_{i}+1}}{\sqrt{\lambda_{i}}} & \text { if } c_{i-1}^{\prime}>\lambda_{i-1} \\
1] & \text { if } c_{i-1}^{\prime}=\lambda_{i-1}
\end{array}, i \geq 1 .\right.
\end{aligned}
$$

Then the expansion in unitary continued fractions giving the best approximations of type 1 reads:

(10)

$$
\xi=a_{0}+[\underbrace{\overline{\varepsilon_{i} \bar{a}_{i}+1 \sqrt{1}+\underline{-1} \sqrt{2}+\cdots+\underline{-1} \sqrt{2}}}_{c_{i}^{\prime}-\lambda_{i}+1}]_{i \geq 1} .
$$

We know exactly which elements of the Hurwitz sequence are the best approximations of type 1 of $\xi$. With the notations of "Intermediate convergents" and "Sequences of Approximations", those are $a_{0}$ and the fractions $\frac{p_{c_{0}^{\prime}+\cdots+c_{i-1}^{\prime}+\lambda}}{q_{c_{0}^{\prime}+\cdots+c_{i-1}^{\prime}+\lambda}^{\prime}}$ with $\lambda=\lambda_{i}, \ldots, c_{i}^{\prime}$ and $i \geq 1$. Therefore it suffices to eliminate the superfluous convergents between $\frac{p_{c_{0}^{\prime}+\cdots+c_{i-1}^{\prime}}}{a_{c_{0}^{\prime}+\cdots+c_{i-1}^{\prime}}}$ and $\frac{p_{c_{0}^{\prime}+\cdots+c_{i-1}^{\prime}+\lambda_{i}}}{a_{c_{0}^{\prime}+\cdots+c_{i-1}^{\prime}+\lambda_{i}}}$. One checks in the example presented above that the expansions in continued fractions of best approximations of type 1 do have the structure described in formula (10). The delicate point consists of checking the condition in the definition of $\lambda_{i}$ when $c_{i}^{\prime}$ is even; this is what the algorithm $B A C F$ described at the end of "The zoo of continued fraction expansions" does automatically.

\section{References}

1. J. Goldman, Hurwitz sequences, the Farey process and general continued fractions, Adv. in Math., 72/2, 1988, 239-260.

2. G. H. HARDY and E. M. WRIGHT, An Introduction to the Theory of Numbers, 5th edition, Oxford Univ. Press, 1979.

3. A. Hurwitz, Über eine besondere Art der KettenbruchEntwicklung reeller Grössen, Acta Math., 12, 1889, 367405; and Oeuvres, tome II, pp. 84-128.

4. A. HuRwitz, Über die angenäherte Darstellung der Zahlen durch rationale Brüche, Math. Ann., 14, 1894, 417-436; and Oeuvres, tome II, pp. 137-156.

5. T. E. MCKINNEY, Concerning a certain type of continued fractions depending on a variable parameter, Amer. J. Math., 29/3, 1907, 213-278.

6. O. Perron, Die Lehre von den Kettenbrüchen, 2nd edition, Chelsea Publ. Company, New York, 1930.

7. I. RICHARDS, Continued fractions without tears, Math. Magazine, 54/4, 1981, 163-171.

\section{Fields Institute Director Search}

The Fields Institute for Research in Mathematical Sciences invites applications or nominations for the position of Director for a three- to five-year term beginning July 1, 2013 (once renewable).

The Fields Institute is an independent research institute located on the downtown campus of the University of Toronto. The Institute's mission is to advance research and communication in the mathematical sciences. With 3000 registered annual participants from around the world, its programs bring together researchers and students, commercial and industrial users, and an interested public. See www.fields.utoronto.ca.

Candidates should be researchers in the mathematical sciences with high international stature, strong interpersonal and administrative skills, and an interest in developing the activities of the Fields Institute.

A letter of application addressing the qualities above, together with a $\mathrm{CV}$ and names of three references should be sent to directorsearch@fields.utoronto.ca. Expressions of interest or nominations may also be sent to this address.

Applications or nominations will be considered until the position is filled, although the Search Committee will begin

\begin{tabular}{|c|c|c|}
\hline ह & $\begin{array}{l}\text { Wavelets } \\
\text { A Concise Guide } \\
\text { Amir-Homayoon Najmi } \\
\text { Najmi's introduction to }\end{array}$ & $\begin{array}{l}\text { Math Goes to } \\
\text { the Movies } \\
\text { Burkard Polster } \\
\text { and Marty Ross }\end{array}$ \\
\hline 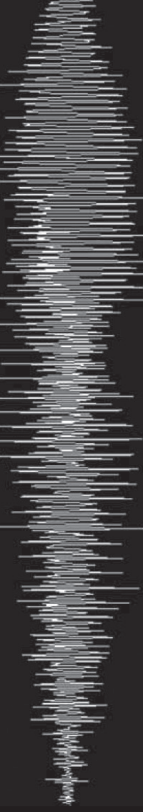 & $\begin{array}{l}\text { wavelet theory explains } \\
\text { this mathematical concept } \\
\text { clearly and succinctly. } \\
\$ 45.00 \text { paperback } \\
\text { Noncommutative } \\
\text { Geometry, } \\
\text { Arithmetic, and } \\
\text { Related Topics } \\
\text { Proceedings of the } \\
\text { Twenty-First Meeting } \\
\text { of the Japan-U.S. } \\
\text { Mathematics Institute } \\
\text { edited by } \\
\text { Caterina Consani } \\
\text { and Alain Connes } \\
\text { This collection of essays by } \\
\text { some of the world's lead- } \\
\text { ing scholars in mathemat- } \\
\text { ics presents innovative } \\
\text { work at the intersection of } \\
\text { noncommutative geom- } \\
\text { etry and number theory. } \\
\$ 85.00 \text { hardcover }\end{array}$ & $\begin{array}{l}\text { Polster and Ross pored } \\
\text { through the cinematic } \\
\text { calculus to create this } \\
\text { entertaining survey of the } \\
\text { quirky, fun, and beautiful } \\
\text { mathematics to be found } \\
\text { on the big screen. } \\
\$ 35.00 \text { paperback } \\
\text { Mathematical } \\
\text { Expeditions } \\
\text { Exploring Word Problems } \\
\text { across the Ages } \\
\text { Frank J. Swetz } \\
\text { "Swetz's choice of } \\
\text { problems is diverse and } \\
\text { insightful. They provide a } \\
\text { lively and engaging way to } \\
\text { incorporate history into a } \\
\text { mathematics course." } \\
\text {-Amy Shell-Gellasch, } \\
\text { Beloit College } \\
\$ 30.00 \text { paperback }\end{array}$ \\
\hline
\end{tabular}
discussions in June 2012. Women and members of underrepresented groups are encouraged to apply. 